\title{
A Study on 13 Cases of Bone and Soft Tissue Sarcomas in the Head and Neck
}

\author{
Shinya Agena, Takayuki Uehara, Yukashi Yamashita, Sen Matayoshi, \\ Masahiro Hasegawa, Hiroyuki Maeda and Mikio Suzuki
}

\begin{abstract}
Since bone and soft tissue sarcomas arising from the head and neck region are very rare, the standard treatment protocol has yet to be established. In the present study, 13 cases with bone and soft tissue sarcomas treated from 2007 to 2014 were analyzed. The mean period from initial visit to final pathological diagnosis was approximately 3 weeks, and 38.5\% of the cases needed several biopsies for an accurate diagnosis. Death occurred early for those cases in which more than 40 days was needed to obtain the histological diagnosis. The 3-year overall survival in 13 cases was $35.2 \%$. As the cause of death was uncontrolled local lesion, the locoregional control was more important than distant metastasis control for the prognosis. Tumor location, TNM stage, and histological grade affected the prognosis of the sarcomas. Radiation therapy including heavy ion and proton beam radiotherapy was not so effective as the primary treatment, as was the case with salvage treatment of sarcomas in the head and neck. Although the primary treatment of sarcomas was surgical treatment with an adequate margin, an efficient treatment protocol should be established to obtain a better prognosis.
\end{abstract}

Keywords : bone and soft tissue sarcomas, head and neck, radiation therapy, prognostic factor, FNCLCC classification

\section{References}

1) Kraus DH, Dubner S, Harrison LB, et al. : Prognostic factors for recurrence and survival in head and neck soft tissue sarcomas. Cancer 74: 697-702, 1994.

2) de Bree R, van der Valk P, Kuik DJ, et al. : Prognostic factors in adult soft tissue sarcomas of the head and neck: a singlecentre experience. Oral Oncol 42: 703-709, 2006.

3) de Bree R, van der Waal I, de Bree E, et al. : Management of adult soft tissue sarcomas of the head and neck. Oral Oncol 46: 786-790, 2010.

4) Le QT, Fu KK, Kroll S, et al. : Prognostic factors in adult softtissue sarcomas of the head and neck. Int J Radiat Oncol Biol Phys 37: 975-984, 1997.

5) Dudhat SB, Mistry RC, Varughese T, et al. : Prognostic factors in head and neck soft tissue sarcomas. Cancer 89: 868-872, 2000.

6) Sturgis EM and Potter BO : Sarcomas of the head and neck region. Curr Opin Oncol 15: 239-252, 2003.

7) Mattavelli D, Miceli R, Radaelli S, et al. : Head and neck soft tissue sarcomas: prognostic factors and outcome in a series of patients treated at a single institution. Ann Oncol 24: 21812189, 2013.

8) 石川征司, 門田伸也, 滝下照章, 他 : 頭頸部領域に発生し た軟部肉腫症例の検討. 頭頸部癌 35: 421-426, 2009.

9）道場隆博，竹中幸則，曺 弘規，他：頭頸部原発成人軟部 肉腫症例の検討。日耳鼻会報 116: 154-160, 2013.

10) O’Sullivan B, Chung P, Euler C, et al. : Soft tissue sarcoma. Clinical radiation oncology 2nd (ed by Gunderson LL and Tepper JE). pp 1519-1546, Churchill Livingstone, Philadelphia, 2007.

11) Trojani M, Contesso G, Coindre JM, et al. : Soft-tissue sarcomas of adults; study of pathological prognostic variables and definition of a histopathological grading system. Int J Cancer 33: 37-42, 1984 .

12) Wanebo HJ, Koness RJ, MacFarlane JK, et al. : Head and neck sarcoma: report of the Head and Neck Sarcoma Registry. Society of Head and Neck Surgeons Committee on Research. Head Neck 14: 1-7, 1992.

13）日本整形外科学会, 骨・軟部腫瘍委員会 : 骨 - 軟部肉腫切 除縁評価法．金原出版，東京，1989。
Department of Otorhinolaryngology, Head and Neck Surgery, University of the Ryukyus
Corresponding Author Address : Shinya Agena

harugen3@yahoo.co.jp 
14) González-González R, Bologna-Molina R, Molina-Frechero N, et al. : Prognostic factors and treatment strategies for adult head and neck soft tissue sarcoma. Int J Oral Maxillofac Surg 41: 569-575, 2012.

15) Crist WM, Anderson JR, Meza JL, et al. : Intergroup rhabdomyosarcoma study-IV: results for patients with nonmetastatic disease. J Clin Oncol 19: 3091-3102, 2001.
16) Pervaiz N, Colterjohn N, Farrokhyar F, et al. : A systematic meta-analysis of randomized controlled trials of adjuvant chemotherapy for localized resectable soft-tissue sarcoma. Cancer 113: 573-581, 2008.

17) Kamada T, Tsujii H, Tsuji H, et al. : Efficacy and safety of carbon ion radiotherapy in bone and soft tissue sarcomas. J Clin Oncol 20: 4466-4471, 2002.

Primary site and pathology of the 13 cases

Sinonasal sarcoma accounted for $46 \%$ of the cases.

\begin{tabular}{|c|c|c|c|c|c|c|c|}
\hline \multicolumn{8}{|c|}{ Site } \\
\hline & & Face & Neck & Oral/oropharynx & Sinonasal cavity & Larynx & Middle ear \\
\hline \multicolumn{8}{|l|}{ Pathology } \\
\hline Rhabdomyosarcoma & 3 & & 1 (Submandibular region) & 1 (Tongue) & 1 (Sinus) & & \\
\hline Undifferentiated pleomorphic sarcoma & 2 & & & 1 (Soft palate) & & 1 & \\
\hline Osteosarcoma & 2 & & & & 2 (Sinus) & & \\
\hline Leiomyosarcoma & 2 & & & & 2 (Sinus, Nasal cavity) & & \\
\hline Synovial sarcoma & 1 & & & & 1 (Nasal cavity) & & \\
\hline Angiosarcoma & 1 & & & & & & 1 \\
\hline Unclassified pleomorphic sarcoma & 2 & 1 (Buccal region) & & 1 (Oral floor) & & & \\
\hline Total & 13 & 1 & 1 & 3 & $6(46 \%)$ & 1 & 1 \\
\hline
\end{tabular}

Summary of 13 cases in relation to the interval from the first visit to the diseases diagnosis, number of biopsies needed to obtain the pathological diagnosis, tumor size, presence/absence of lymph node metastasis, AJCC stage classification, and FNCLCC classification

More than 40 days were required before a pathological diagnosis could be obtained in the three cases. Tumor size of less than $5 \mathrm{~cm}$ are two cases. Three cases are disease-free survival.

\begin{tabular}{|c|c|c|c|c|c|c|c|c|c|c|c|c|c|c|c|c|}
\hline \multirow[b]{2}{*}{ Case } & \multirow[b]{2}{*}{ Sex } & \multirow[b]{2}{*}{ Age } & \multirow[b]{2}{*}{ Site } & \multirow[b]{2}{*}{ Pathology } & \multirow{2}{*}{$\begin{array}{c}\text { Pathological } \\
\text { diagnosis period }\end{array}$} & \multirow{2}{*}{$\begin{array}{l}\text { Count } \\
\text { biopsy }\end{array}$} & \multirow{2}{*}{$\begin{array}{l}\text { Tumor diameter } \\
\text { Major axis } \\
\times \text { minor axis }\end{array}$} & \multirow{2}{*}{$\begin{array}{l}\text { Lymph } \\
\text { node } \\
\text { metastasis }\end{array}$} & \multicolumn{3}{|c|}{ AJCC Stage } & \multicolumn{5}{|c|}{ FNCLCC Classification } \\
\hline & & & & & & & & & $\mathrm{T}$ & $\mathrm{N}$ & M & Stage & $\begin{array}{c}\text { Tumor } \\
\text { differentiation }\end{array}$ & $\begin{array}{c}\text { Mitosis } \\
\text { count }\end{array}$ & $\begin{array}{c}\text { Tumor } \\
\text { necrosis }\end{array}$ & Grade \\
\hline 1 & $\mathrm{~F}$ & 55 & Sinus & Rhabdomyosarcoma & 20 & 1 & $5.3 \times 2.2$ & + & $\mathrm{T} 2 \mathrm{~b}$ & 1 & 0 & IV & 3 & 3 & 0 & 3 \\
\hline 2 & M & 9 & $\begin{array}{l}\text { Submandibular } \\
\text { region }\end{array}$ & Rhabdomyosarcoma & 21 & 1 & $5.7 \times 4.5$ & - & $\mathrm{T} 2 \mathrm{~b}$ & 0 & 0 & III & 3 & 3 & 0 & 3 \\
\hline 3 & M & 9 & Tongue & Rhabdomyosarcoma & 22 & 1 & $5.1 \times 3.2$ & - & $\mathrm{T} 2 \mathrm{~b}$ & 0 & 0 & III & 3 & 3 & 2 & 3 \\
\hline 4 & $\mathrm{~F}$ & 49 & Soft palate & $\begin{array}{l}\text { Undifferentiated } \\
\text { pleomorphic sarcoma }\end{array}$ & 165 & 2 & $5.1 \times 4.9$ & - & $\mathrm{T} 2 \mathrm{~b}$ & 0 & 0 & III & 3 & 1 & 0 & 2 \\
\hline 5 & M & 77 & Larynx & $\begin{array}{l}\text { Undifferentiated } \\
\text { pleomorphic sarcoma }\end{array}$ & 23 & 1 & $1.0 \times 1.0$ & - & Tla & 0 & 0 & I & 2 & 1 & 0 & 1 \\
\hline 6 & M & 22 & Maxillary sinus & Osteosarcoma & 27 & 1 & $7.6 \times 5.5$ & - & $\mathrm{T} 2 \mathrm{~b}$ & 0 & 0 & III & 3 & 1 & 0 & 2 \\
\hline 7 & M & 34 & Maxillary sinus & Osteosarcoma & 22 & 2 & $6.0 \times 3.1$ & - & $\mathrm{T} 2 \mathrm{~b}$ & 0 & 0 & III & 2 & 1 & 2 & 2 \\
\hline 9 & $\mathrm{~F}$ & 59 & Nasal cavity & Leiomyosarcoma & 22 & 1 & $3.6 \times 1.9$ & - & T1b & 0 & 0 & I & 2 & 1 & 0 & 1 \\
\hline 10 & M & 57 & Nasal cavity & Synovial sarcoma & 90 & 2 & $7.6 \times 3.9$ & - & $\mathrm{T} 2 \mathrm{~b}$ & 0 & 0 & III & 3 & 1 & 1 & 2 \\
\hline 11 & M & 72 & Middle ear & Angiosarcoma & 41 & 2 & $5.2 \times 4.0$ & - & $\mathrm{T} 2 \mathrm{~b}$ & 0 & 0 & I & 2 & 1 & 0 & 1 \\
\hline 12 & M & 38 & Buccal region & $\begin{array}{l}\text { Unclassiffied } \\
\text { pleomorphic sarcoma }\end{array}$ & 33 & 2 & $5.3 \times 4.0$ & - & $\mathrm{T} 2 \mathrm{~b}$ & 0 & 0 & III & 3 & 1 & 0 & 2 \\
\hline 13 & M & 45 & Oral floor & $\begin{array}{l}\text { Unclassiffied } \\
\text { pleomorphic sarcoma }\end{array}$ & 24 & 1 & $8.5 \times 5.0$ & - & $\mathrm{T} 2 \mathrm{~b}$ & 0 & 0 & III & 3 & 1 & 1 & 2 \\
\hline
\end{tabular}


Treatment and prognosis of the 13 cases

Only one case received palliative treatment, while the remaining 12 cases received curative treatment. Three of the four cases in whom surgery was performed first remain alive. All of the cases who received chemotherapy prior to surgery or radiotherapy died. In 10 of the cases in which surgery was performed, the surgical margins were negative, however, 6 of these cases $(60 \%)$ died of local recurrence.

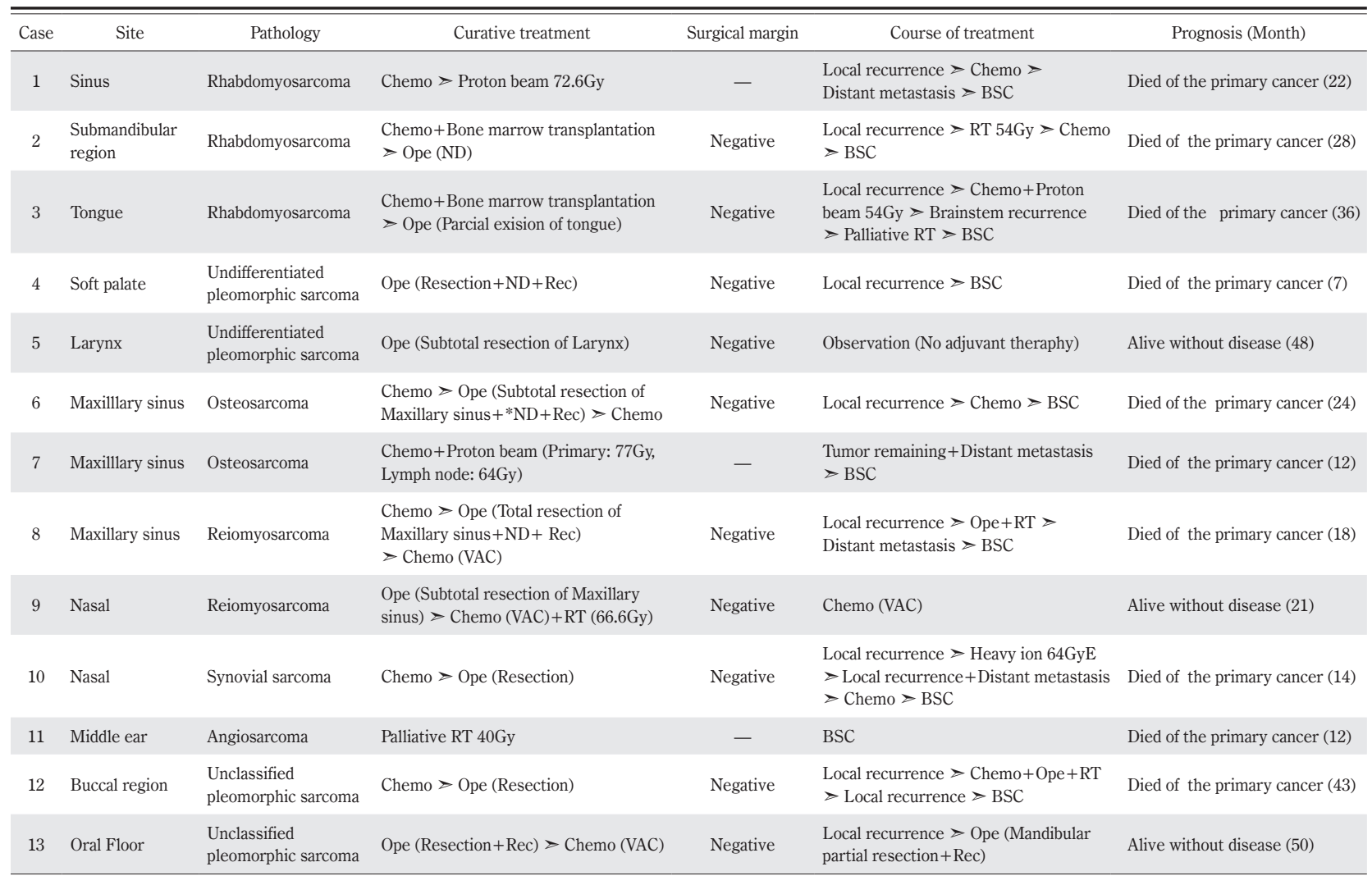

BSC: Best supportive care; Chemo: Chemotherapy, Gy: Gray; ND: Neck Dissection; Ope: Operation; RT: Radiation, VAC: Vincristine/ActinomycinD/Cyclophosphamide; Rec: Reconstruction 